\title{
THE FISH EIRG IN RESPONSE TO DIFFERENT TYPES OF ILLUMINATION
}

\author{
TSUNEO TOMITA, TSUNEO TOSAKA, \\ KOSUKE WATANABE AND YUKIO SATO* \\ Department of Physiology, Tokyo Women's Medical College, Ushigome, Tokyo, and \\ Department of Physiology, Keio University School of Medicine, Yotsuya, Tokyo
}

In a preceding paper (11), the cone action potential described by Svaetichin $(8,9)$, the characteristic response to illumination from within the fish retina, was reinvestigated by one of the authors, using a coaxial pencil-type microelectrode $(10,14)$. Its origin was concluded to be proximal to the visual cell layer. An alternative term, the fish EIRG, was thus given to the same potential change. The present paper will deal with some properties of this response as revealed by different types of illumination.

\section{METHOD}

The excised eye of Cyprinus auratus was opened and mounted on a silver plate which served as an indifferent electrode. In some experiments, however, the retina was detached from the pigment epithelium and placed on the silver plate covered by a piece of black paper soaked with aqueous humor from the same eye. The black paper bed was used to minimize the scattering of light.

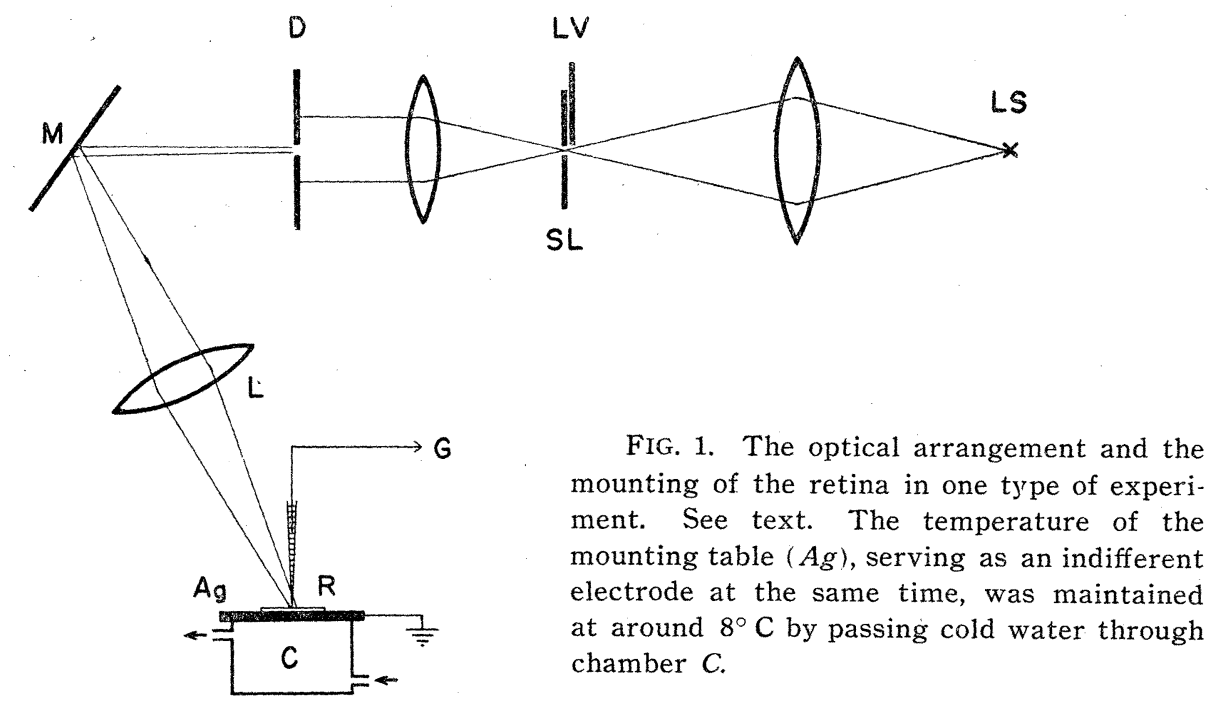

Received for publication December 2, 1957.

* 冨田恒男, 登抜恒夫, 渡辺宏助, 作藤幸男 
The area effect was studied with the optical arrangement shown in fig. 1. Light from a single coil tungsten lamp $(L S)$ was focused on slit $S L$. The light valve $(L V)$ in front of the slit was remotely operated and synchronized with the sweep of the oscilloscope beam. A diaphragm $(D)$ with a changeable aperture in the center was inserted into the light path and imaged on the retina by an anastigmatic lens $(L)$.

In another type of experiment, the diaphragm $D$ was replaced by a metal plate as shown in fig. 2. The plate (shadowed in the figure) was provided with a slit $(S)$, an edge $(E)$, and a wire stretched between two arms $(W)$. An image of any one of them could be focused on the retina. The image of either the slit or wire appeared as a light or dark stripe about $0.2 \mathrm{~mm}$. wide on the retina.

In still another type of experiment, the "spectrum sweep method" which was originally used for investigating color responses of the frog retina (12) was applied. Fig. 3 illustrates the optical system used. A spectroscope $(S)$ of Adam-Hilger type served as a monochromator. By rotating its wave-length drum $(D)$, the wave-length of illuminating light could be changed continuously throughout the visible spectrum. However, in order that the change of wave-length per unit time, or $d \lambda / d t$, was as constant as possible, the drum $D$ provided with a point to rotate the prism table was removed altogether and replaced by a mechanism shown in fig. 4 . The rotation of the prism was then achieved by a motion of the point $D p$ at one

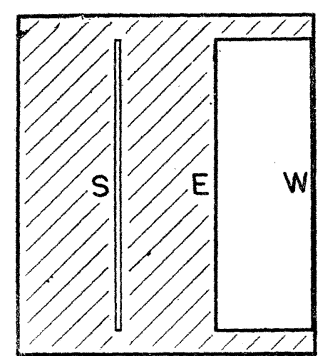

FIG. 2. Diaphragm placed for $D$ in fig. 1 and imaged on the retina. See text. end of the lever $L$. The opposite arm of this lever is

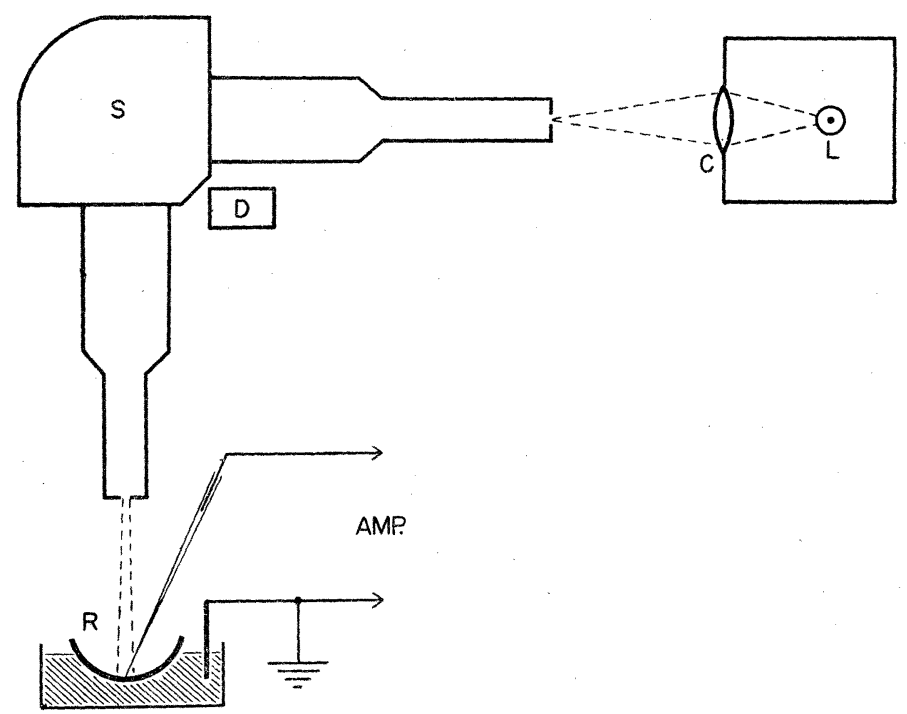

FIG. 3. Optical arrangement for the "spectrum sweep." See text. 


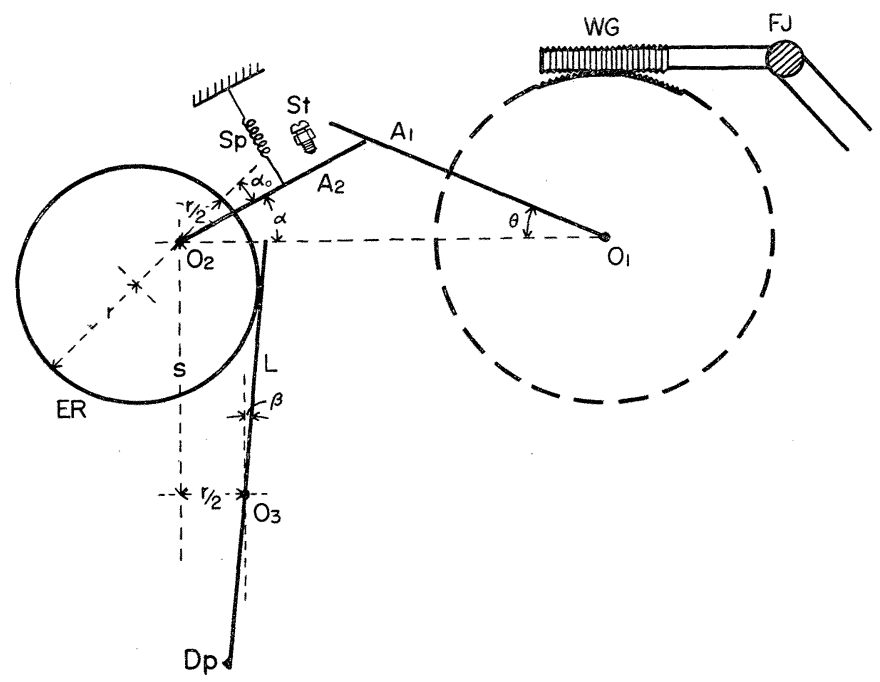

FIG. 4. Scanning mechanism shown by $D$ in fig. 3 . Explanation in text.

in contact with an eccentric gear $(E R)$ whose rotation is controlled by its arm $\left(A_{2}\right)$ sliding along the $\operatorname{arm} A_{1}$ of the wheel $O_{1}$ which is rotated in either direction at a constant angular velocity by means of a motor driven worm-gear $(W G)$. The rotation, however, was limited between two certain angular positions of the arm $A_{1}$, beyond which an electromagnetically operated clutch was opened automatically to cut off the driving force. The motion of the point $D p$ is determined primarily by

$$
d \beta / d t=d \theta / d t \times d \alpha / d \theta \times d \beta / d \alpha^{*} .
$$

Each rotation of the rod connecting the worm-gear with a synchronous motor by way of flexible joint ( $F J$ ) marked a pip on the record (cf. figs. 8-10). As the rod was rotated once in every 0.4 second, the pips marked this time interval. Besides, they indicated definite wave-lengths. The pip number/wavelength relation was plotted in fig. 5 by the aid of several conspicuous spectra of the mercury lamp. The linearity was not a very satisfactory one, but the adgustments done empirically by changing $\alpha_{0}$ and the arm ratio of the lever $L$ in fig. 4 needed a patience and had to be compromised. In another attempt to obtain an equal energy spectrum throughout the visible range, a diaphragm was designed that could control automatically the light flux into the monochromator, but it was not used in the present experiment as it made the intensity of illumination too weak.

* Under the conditions specified in the text and in fig. $4, d \theta / d t=$ const.,

$$
\begin{gathered}
d x / d \theta=\frac{2 \cos \theta-\sqrt{4 \cos ^{2} \theta-3}}{\sqrt{4 \cos ^{2} \theta-3}} \text {, and } d \beta / d \alpha=\frac{1}{n^{2}-2 p}\left[\frac{2\left\{\sin \left(\alpha+\alpha_{0}\right)+n \cos \left(\alpha+\alpha_{0}\right)\right\}}{\left.\sqrt{n^{2}-2 p-4}+p\right]},\right. \\
\text { where } n=\frac{s}{r / 2}, \text { and } p=n \sin \left(\alpha+\alpha_{0}\right)+\cos \left(\alpha+\alpha_{0}\right)-1
\end{gathered}
$$




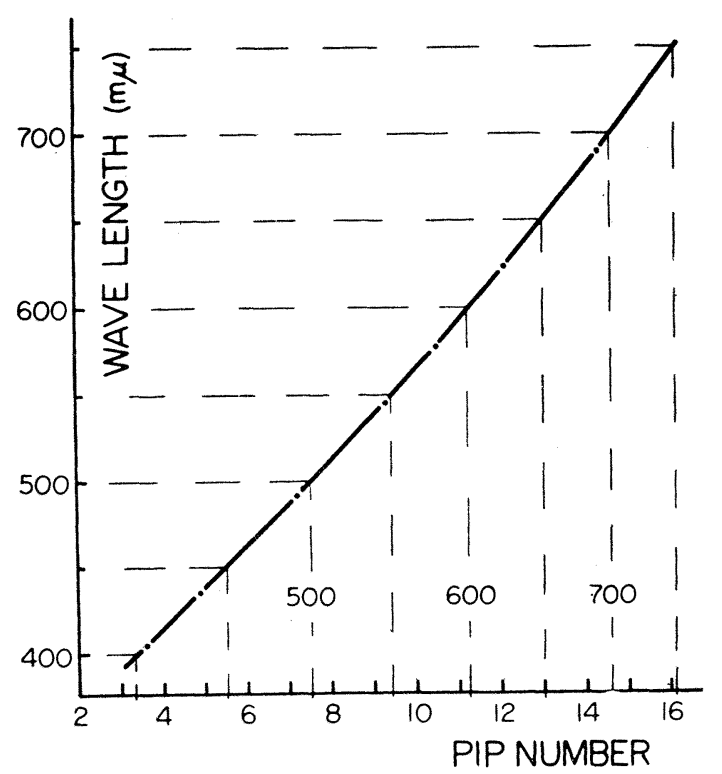

FIG. 5. Calibration curve of wave-length against pip-number.

\section{RESULTS}

\section{Effect of illumination patterns upon the response}

Fig. 6 illustrates an example of one series of recordings of the fish EIRG with a microelectrode placed in the center of a light spot of various diameters but of constant intensity (about 200 Lux). In these recordings, electrode insertion was made from the receptor side of isolated retina, and a background illumination of about $20 \mathrm{Lux}$ was applied after Brindley $(3,4)$ to minimize the effect of scattered light. As is clearly seen in the figure, the response became smaller as the illumination area was decreased. In the record $E$ which was obtained by a light spot of $0.2 \mathrm{~mm}$. across, the response was hardly detectable, making a strong contrast to the focal negative potential change from within
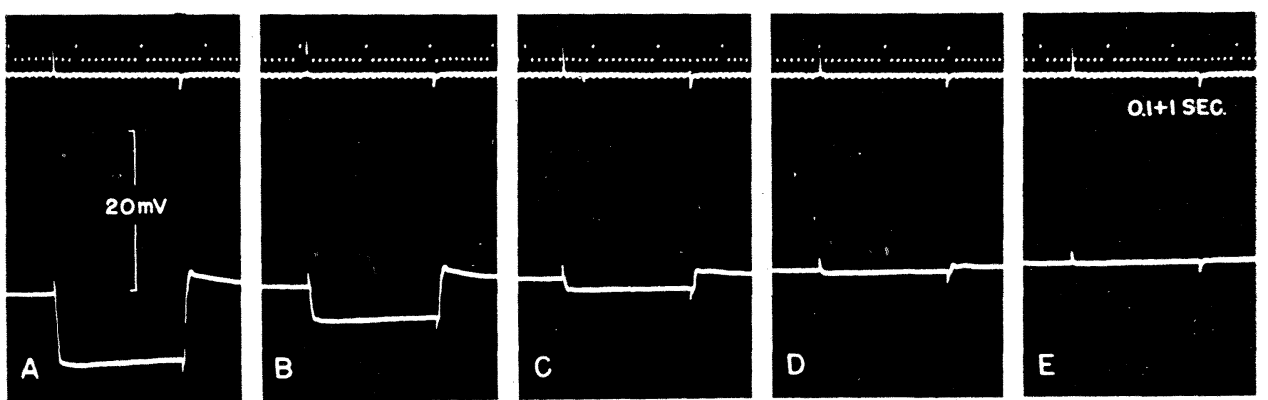

FIG. 6. A series of recordings of the fish EIRG in response to illumination of various areas, whose diameters on the retina were; $3.2 \mathrm{~mm}$. in $A, 1.6 \mathrm{~mm}$. in $B .0 .8$ $\mathrm{mm}$. in $C, 0.4 \mathrm{~mm}$. in $D$, and $0.2 \mathrm{~mm}$. in $E$. 
the frog retina that was found by Tomita et al. $(12,13)$ to be very sensitive to microillumination and confirmed by Brindley (4). Evidently, activities of neighboring receptors take part in generation of the fish EIRG. In this respect, the fish EIRG resembles the ERG which was reinvestigated recently on the cat by Wirth and Zetterström (16) who excluded the scattered light by applying a Perspex cone in front of the retina. Brindley also demonstrated a similar behavior of the frog ERG, though he is inclined to account for the result more physically than physiologically $(2,3)$.

Further evidence that the fish EIRG is due to the summation over a large receptive field is the fact that, when the edge of a shadow was moved across the site of recording, no abrupt change occurred in the response amplitude. Fig. 7 illustrates responses to three types of illumination with the diaphragm shown in fig. 2. The heading on each row indicates the portion of the diaphragm used for recording. The upper trace of $S a$ was obtained with an electrode in a light stripe, the image of the slit on the retina. In $S b$ in which the light stripe was $0.34 \mathrm{~mm}$. away from the electrode, response was still visible though smaller in size. Sc was obtained by even illumination on the whole retina. The lower trace in $S a$, taken under the same conditions as the upper one but after recording $\mathrm{Sc}$, served as a control at the end of the experiment.

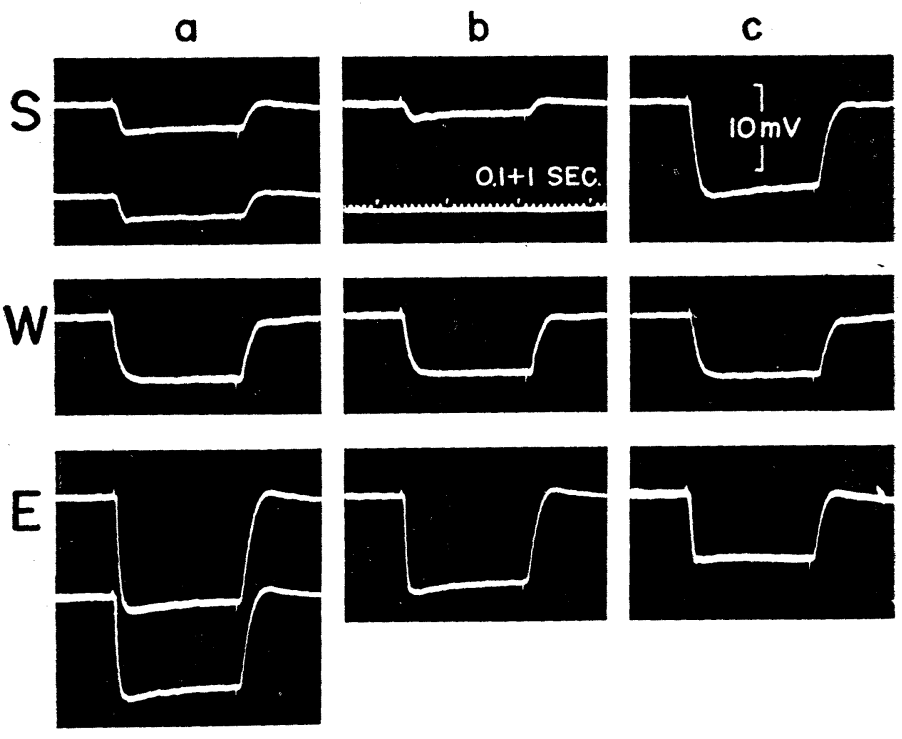

FIG. 7. Responses to illumination by means of the diaphragm shown in fig. 2 . See text.

The records shown in the middle row of fig. 7 were taken when a dark stripe or the image of the wire $W$ was $0.34 \mathrm{~mm}$. away from the electrode to the left side $(W a)$, just on the electrode $(W b)$, and $0.34 \mathrm{~mm}$. away from the electrode to the opposite side $(W c)$. The effect of the shadow upon the response was so small (about $5 \%$ ) that inspection of the oscilloscope screen failed to 
detect it. For the recordings at the bottom, the edge of the diaphragm was used. In $E a$, the electrode was in the light area but $0.34 \mathrm{~mm}$. away from the border of light and dark. The border was then moved just onto the site of recording for $E b$, and $0.34 \mathrm{~mm}$. further to bring the electrode into the dark area for $E c$. The lower trace in $E a$ represents a control taken after the above recordings. While the border was moved across the electrode, the response amplitude changed somewhat but only very gradually.

\section{Response curves to the spectrum sweep}

Svaetichin discovered that the fish EIRG from different retinal regions responds differently to light of different wave-lengths. He classified the spectral response curves into three types; $L, R / G$ and $B / Y$. Motokawa, Oikawa and Tasaki (7) who worked independently on the carp also obtained similar response curves but called them differently; $N$-type for Svaetichin's $L$-type, and $A$-type for $B / Y$-type. No $R / G$-type has been found in the carp. In these works, the retina was illuminated successively by light whose wave-length was changed stepwise.

In the present experiment with the spectrum sweep method, the wave-length was changed continuously through the visible spectrum in about 5 seconds, and this enabled very rapid successive recordings from various regions of the same retina. The electrode insertion and illumination were made from the vitreous side of the opened excised eye of Cyprinus auratus. So far as the excised eye was concerned, this material was distinguished by its very small blood content compared with that of Cyprinus carpio. The influence of the blood upon the incident light appeared to be negligibly small in this retina. The monochromatic light from the scanning monochromator made a patch of $1.5 \mathrm{~mm} \times 3.0$ $\mathrm{mm}$. on the retina. A recording electrode was situated in its center.

The records in figs. 8 through 10 were picked out of a number of those obtained from one and the same retina. In each recording, the spectrum was swept from the blue side to red side (left column $B \rightarrow R$ ) and then in the opposite direction (right column $R \rightarrow B$ ). Responses in pairs were nearly symmetrical, while the responses from different regions of the eye were of various shapes. In fig. $9 b$, the $R \rightarrow B$ response was inverted photographically and is shown below the $B \rightarrow R$ response. Two curves are shifted a little due to response delay.

Fig. $8 a$ and $b$ represent simple forms of response with no discernible hump anywhere. Their peaks, however, are at different wave-lengths. In fig. $8 c$, there is a small hump near the red end, and in fig. $9 a$ an additional hump near the blue end. The peaks of fig. $9 b$ and $c$ are shifted more towards red side and the hump on the blue side is larger. In all the above six response curves which appear to belong to Svaetichin's $L$-type, the potential level at the red end was lower than at the blue end, indicating that the units were still sensitive to wavelengths longer than $750 \mathrm{~m} \mu$. A response to turning on and off the light at the red end is shown in the inset above fig. $8 c$.

Three response curves illustrated in fig. 10 may correspond to Svaetichin's $B / Y$-type. The curves $10 a$ and $b$ are much similar, but a small dip near the red end in the former suggests a triphasic response such as demonstrated by 


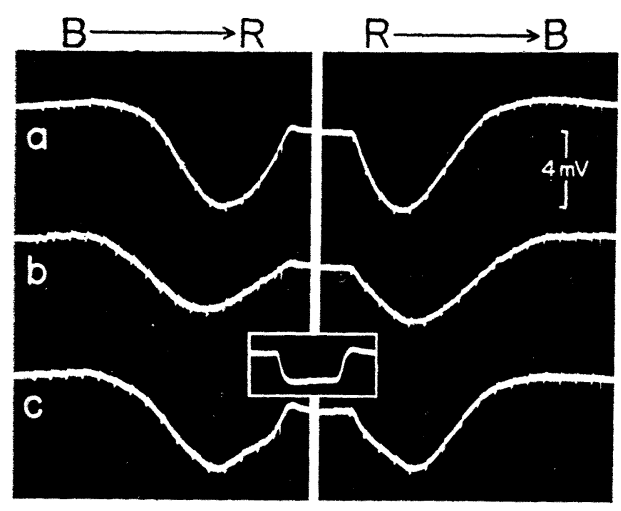

FIG. 8

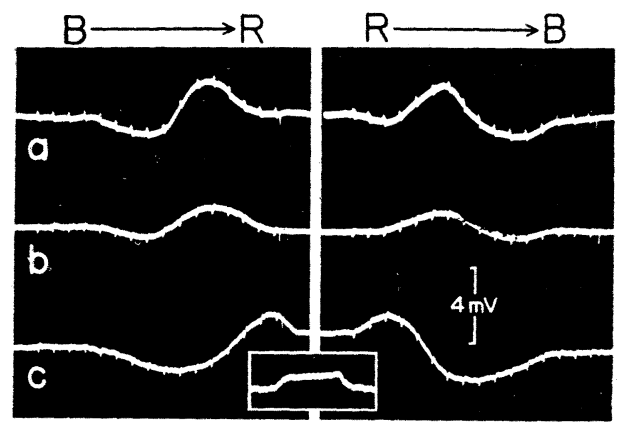

FIG. 10

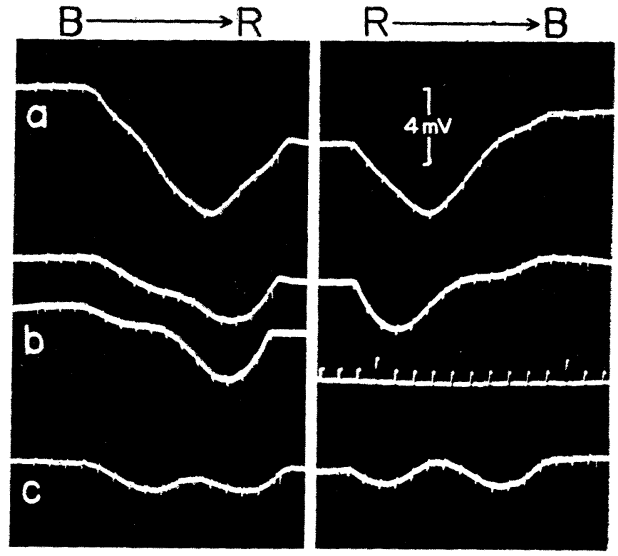

FIG. 9

FIGS. 8, 9 and 10. Some of different response curves obtained from one and the same retina with the spectrum sweep method. See text. Time marking: $0.5+$ 5 seconds.

Motokawa et al. In the curve $10 c$, the peak is shifted more towards red side and a positive deflection persisted at the red end as shown in the inset.

All the other response curves obtained appeared to resemble some one of the illustrated curves, but their peaks and humps were at various positions in the spectrum. It should be noticed, however, that our curves differ considerably from the spectral response curves to be obtained with equal energy spectrum, such as those of Svaetichin and Motokawa et al. For a rough estimation of the deviations, two curves are shown in fig. 11 which were plotted from fig. $9 b$ and $10 c$ by means of the following procedures: 1) $R \rightarrow B$ response was inverted and superimposed on $B \rightarrow R$ response (cf. fig. $9 \mathrm{~b}$ ) and their mean curve was drawn graphically. 2) The spectral energy distribution of stimulating light was calculated from the temperature of light source and also from the dispersive power of the monochromator at different wave-lengths. 3) The graphically obtained mean curve was corrected on the assumption that the response amplitude $(A)$ at each wave-length is proportional to square root of light energy (I), or $A=k \sqrt{I}$, provided that $I$ is not very strong. This assumption was based on the $A / I$ relation curve of Svaetichin (8) and also on our own observation. 


\section{DISCUSSION}

1. The fish EIRG is the kind of response that one would expect to record from the receptors. Similar slow action potentials in the invertebrate retinae have been suggested by many investigators $(1,5,6,15)$ to arise from a very peripheral structure. Nevertheless, the experiment in a preceding paper in which the depth of the response in the fish retina was measured appeared to show its more proximal origin. The effect of area illuminated and of shading observed in the present experiment also suggests that the response is due to the activities of second or higher order of neurons onto which a number of receptors converge directly or indirectly. If a receptor cell were the real origin, the response would mainly, if not wholly, be determined by the light falling directly upon it whether or not its neighbors are being illuminated.

2. On the belief that the fish EIRG originates in the cones, Svaetichin attributed the several elevations in his $L$-type responses and the opposite deflections in $R / G$ - and $B / Y$-type of responses to different photosensitive substances contained in single cones and to functioning of twin-cones respectively. According to our present viewpoint, on the other hand, they may be the results of integration by convergence of a number of receptors whose individual responses to colored light could be much simpler.

3. Since the fish EIRG was found to be detectable with microelectrodes of $5 \mu$ or more diameter as well, question has been raised concerning its intracellular nature (11). On the other hand, in apparent support of the conventional view was the fact that the response becomes small much faster at the very site of recording, due probably to a mechanical injury by the electrode, while the activities in its close vicinity, as explored later by the same electrode, are well maintained. The different response curves to colored light from different regions in the same retina (figs. 8-10) may also suggest that the recordings were made from within some kind of compartments isolated electrically from each other. A histological approach combined with microelectrode techniques will be the key to a solution of this problem. 
SUMMARY

1. The fish EIRG (Svaetichin's cone action potential) was investigated on Cyprinus auratus with several types of illumination.

2. The response amplitude depended strongly upon the area illuminated, becoming very small when the diameter of light spot was decreased to $0.2 \mathrm{~mm}$.

3. During passage of the border of light and dark across the site of recording, no abrupt change occurred in the response amplitude.

4. Illumination by monochromatic light whose wave-length was changing continuously through the visible spectrum produced various response curves from different regions of the same retina. The result appeared to confirm Svaetichin and Motokawa et al., but details could not be compared due to the difference in experimental conditions.

5 . Based on the above results, the nature of the response was discussed.

\section{ACKNOWLEDGMENT}

The authors wish to express appreciation to Dr. E. F. MacNichol, Jr., Biophysics Department of Johns Hopkins University, for his valuable suggestions in making this paper.

This work was aided by a grant from the Ministry of Education.

\section{REFERENCES}

1. BERNHARD, C. G. Isolation of retinal and optic ganglion response in the eye of Dytiscus. J. Neurophysiol. 5: 32-48, 1942.

2. BRINDLEY, G. S. The passive electrical properties of the frog's retina, choroid and sclera for radial fields and currents. J. Physiol. 134: 339-352, 1956.

3. BRINDLEY, G. S. The effect on the frog's electroretinogram of varying the amount of retina illuminated. J. Physiol. 134: 353-359, 1956.

4. BRINDLEY, G. S. Responses to illumination recorded by microelectrodes from the frog's retina. J. Physiol. 134: 360-384, 1956.

5. HANAOKA, T. The electric response which seems to be elicited in a unit receptor of simple retina of cray-fish. J. Zool. (in Japan), 59: 226-229, 1950.

6. Miller, W. H. Morphology of Limulus ommatidia. J. Biophys. Biochem. Cytol. 3: 421$428,1957$.

7. MotokAwa, K., OIKAWA, T. AND TASAKI, K. Receptor potential of vertebrate retina. J. Neurophysiol. 20: 186-199, 1957.

8. Svaetichin, G. The cone action potential. Acta Physiol. Scand. 29: Suppl. 106, 565600, 1953.

9. Svaetichin, G. Spectral response curves from single cones. Acta Physiol. Scand. 39: Suppl. 134, 17-46, 1956.

10. Tomita, T. The nature of action potentials in the lateral eye of the horseshoe crab as revealed by simultaneous intra- and extracellular recording. Jap. J. Physiol. 6: 327$340,1956$.

11. Tomita, T. A study on the origin of intraretinal action potential of the Cyprinid fish by means of pencil-type microelectrode. Jap. J. Physiol. 7: 80-85, 1957.

12. Tomita, T., Funaishi, A. And Mizuno, H. Some properties of slow negative component in bullfrog's intraretinal action potential. XIX Int. Physiol. Congr., Abstr. of Communications, pp. 829-830, 1953. 
13. Tomita, T., Mizuno, H. AND IDA, T. Studies on the intraretinal action potential. Part III. Intraretinal negative potential as compared with b-wave in the ERG. Jap. J. Physiol. 2: 171-176, 1952.

14. Tomita, T. AND Torihama, Y. Further study on the intraretinal action potentials and on the site of ERG generation. Jap. J. Physiol. 6: 118 136, 1956.

15. WAterman, T. H. AND Wiersma, C. A. G. The functional relation between retinal cells and optic nerve in Limulus. J. exp. Zool. 126: 59-86, 1954.

16. Wirth, A. AND ZETTERström, B. The effect of area and intensity on size and shape of the ERG. The exclusion of stray light effect. Brit. J. Ophthal, 38: 257-265, 1954. 